\title{
Optimal Generator Portfolio in Day-Ahead Market under Uncertain Carbon Tax Policy
}

\author{
Shengyuan Chen ${ }^{1}$, Ming Zhao ${ }^{2}$ \\ ${ }^{1}$ Department of Mathematics and Statistics, York University, Toronto, Canada \\ ${ }^{2}$ SAS Institute Inc., Cary, USA \\ E-mail: chensy@mathstat.yorku.ca,Ming.Zhao@sas.com \\ Received August 26, 2011; revised September 24, 2011; accepted October 12, 2011
}

\begin{abstract}
The global liberalization of energy market and the evolving carbon policy have profound implication on a producer's optimal generator portfolio problem. On one hand, the daily operational flexibility from a wellcomposed generator portfolio enables the producer to implement a more aggressive bidding strategy in the liberalized day-ahead market on a daily basis; on the other hand, the evolving carbon policy demands the long term robustness of a generator portfolio: it should be able to generate stable cash flow under different stages of the evolving carbon tax policy. It is computationally very challenging to incorporate the daily bidding strategy into such a long term generator portfolio study. We overcome the difficulty by a powerful vertical decomposition. The long term uncertainty of carbon tax policy is simulated by scenarios; while the daily electricity price fluctuation with jumps is modeled by a more complicated Markov Regime Switching model. The proposed model provides the senior executives an efficient quantitative tool to select an optimal generator portfolio in the deregulated market under evolving carbon tax policy.
\end{abstract}

Keywords: Carbon Tax, Generator Portfolio, Markov Regime Switching Model, Stochastic Programming, Unit Commitment, Simulation

\section{Introduction}

This paper analyzes the impact of the evolving carbon tax and market deregulation on a thermal power's optimal generator portfolio. In a centralized market, a generator portfolio is optimized towards minimizing production cost under the constraint that the dynamic demand must be satisfied. The optimal generator portfolio problem has been extensively studied under the centralized market environment. Under this environment, an optimal portfolio typically include a significant portion of base load generators and certain amount of peak load capacity. However, in a decentralized market, satisfying demand is not a hard constraint anymore. Instead, a power producer competes in the day-ahead market to maximize their profit. Bidding strategy, rather than satisfying demand, turns out to be a critical component in a power producer's business. Carbon tax also brings a new dimension into the study since a traditional base load generator is not as cost effective as before if the carbon tax imposes a high environment fee. Carbon tax has been piloted in some jurisdictions, however, the timing, stringency and in- struments are evolving. New technologies to reduce carbon foot print of thermal generators have also been invented and must be included in a current study of the optimal generator problem, as well as the scenarios of carbon tax and the bidding strategies.

In a day-ahead market, producers and consumers submit hourly multiple price and quantity pairs, which are used to construct a bidding curves by linear interpolation. We assume that every producer bids all capacity in one of more pairs at increasing prices of its choices. The ISO (Independent System Operator) computes the clearing price for each hour based on the submitted production and consumer bids. The market-clearing price is used to pay any accepted production bid and is also the price paid by any accepted demand bid. In such a situation, the profit maximization problem faced by a producer decomposes into independent subproblems for each generator owned by the producer, see [1]. However, the optimal generator portfolio has to be considered in integrity due to two reasons: capital investment is shared by different generators; and characteristics of generators are complementary under different policy and price scenar- 
ios.

We analyze the classical problem under the new environments from the perspective of a single price-taker producer, and we make an important assumption that the producer has no power to alter the market clearing price. This pool-based electricity market has also been assumed in $[2,3]$ as well. In contrast, in a study of market equilibrium, one must assume that the collective effort of all producers will change the market price. [4] makes this assumption and derives an optimal portfolio for the market, instead of an individual producer. Other studies of the optimal generator problem have different focuses, for example, [5] considers a multiple objective model; and [6] put emphasize on a risk adjusted mean return model.

The paper is organized as the followings: Section 2 presents our stochastic optimization model; Section 3 models the bidding strategy of a price-taker producer; Section 4 is devoted to modelling the stochastic process underlying the problem of study, and is divided into three subsections addressing electricity price, natural gas price and carbon tax separately; Section 5 establishes the decomposition theorem, which is the foundation of the proposed methodology. Finally, we conduct a case study in Section 6 to demonstrate the efficiency of our algorithm using real market data from Ontario, Canada.

\section{Stochastic Optimization Model}

We consider the optimal generator portfolio for a long but fixed period consisting of $\bar{D}$ days. Within this planning horizon, we are uncertain about the carbon tax $r$, electricity price $\xi$ and natural gas price $\zeta$. We use a set of scenarios to represent the uncertainty.

$S_{r}$ : set of carbon policy scenarios;

$S_{\zeta}$ : set of natural gas price scenarios;

$S_{\xi}$ : set of electricity price scenarios;

$\eta$ : combined random vector $\eta=[r, \zeta, \xi]$

$S$ : set of scenarios for $\eta: S_{r} \times S_{\zeta} \times S_{\xi}$;

The set of generator technologies and the planning horizon is described by the following notations:

$I:$ set of generator types;

$k_{i}$ : capital cost of generator $i \in I$;

$D$ : set of days in the planning horizon;

$M$ : set of months in the planning horizon;

$M_{m}$ : set of days in the $m$ th month;

Let $x_{i}$ be the percentage of investment for the generator type $i$, then the vector $x$ represents the generator portfolio. The portfolio $x$ is subject to the following budget constraint:

$$
\sum_{i \in I} x_{i} \leq 1
$$

We request that the generator portfolio must be able to generate a minimum monthly cash flow $\bar{f}_{m}$. Let

$f_{d}^{s}(x)$ : cash flow on day $d$ from a generator portfolio $x$ under a scenario $s$;

$f_{d i}^{s}\left(x_{i}\right)$ : cash flow on day $d$ from the generator $i$ in a portfolio $x$ under the scenario $s$, then

$$
\sum_{d \in M_{m}} f_{d}^{s}(x) \geq \bar{f}_{m} \quad \forall s \in S, \quad m \in M,
$$

imposes this single-sided cash flow risk control. A minimum cash flow is often required by many financial institutions a indicator that its customers are at good financial status.

Our goal is to maximize the expected profit of a generator portfolio in the planning horizon. Since this is a long-term planning problem, we discount the future profit to its present value. Using discounting factor $\gamma$, the net present value (NPV) of the cash flow from a portfolio $x$ under a scenario $s$ can be calculated as

$$
N P V^{s}(x)=\sum_{d \in D} \gamma^{d} f_{d}^{s}(x),
$$

Assuming zero salvage value of all generators at end of planning horizon for simplicity, we can model the optimal generator portfolio problem as:

$$
\max _{x} \mathbb{E}_{\eta} \operatorname{NPV}(x, \eta),
$$

where the expectation $\mathbb{E}_{\eta} \operatorname{NPV}(x, \eta)$ is numerically approximated by $\sum_{s \in S} w^{s} \mathrm{NPV}^{s}(x)$ with $S$ scenarios of weight $w^{s}$.

The calculation of $f_{d}$ is complicated by the bidding strategy of the power producer. In the centralized market, one might calculate the revenue as price times the demand. However, in a day-ahead market, a producer submits bids to an independent system operator (ISO) in the previous day, and can only sell the amount indicated on the bidding result returned from the ISO. As a result, it is not only the generator's maximum output rate determines the revenue; the producer's bidding strategy also jointly determines its cashflow. Intuitively, an intelligent bidding strategy can facilitate cashing in a generator's production capability, thermal efficiency and the portfolio's structural flexibility. To quantitatively establish the relation between the bidding strategy and the generator portfolio, i.e., the function $f_{d}(x)$, we explicitly model the optimal bidding strategy of a price-taker producer in the next section.

\section{Model the Bidding Behaviour}

In [7], a producer's bidding strategy is divided into three steps: forecast the next day electricity price; solve a selfscheduling problem; derive a bidding combination from 
the optimal solution of the self-scheduling problem. [7] shows that the bidding strategy achieves a satisfactory performance in a price-taker pool market. We assume the producer will follow the strategy consistently. In this strategy, the producer is also assumed to have a price forecasting model.

$\psi(\eta)$ : bidding strategy as a function of known information;

$\xi^{\text {est }}$ : producer's estimate of the next day electricity price;

$\zeta^{\text {est }}:$ producer's estimate of the next day natural gas price;

$\xi^{\mathrm{com}}$ : market clearing price of the next day electricity;

$p_{h i}^{\text {est }}(x)$ : producer's bidding production plan for generator $i$ for hour $h$;

$p_{h i}^{\text {com }}(x)$ : producer's committed production as required by the bidding result from ISO;

$\zeta^{\text {tru }}$ : next day true natural gas price;

As pointed out in [7], for a price-taker producer, the bidding problem can be decomposed into profit maximization problems for each generator owned by the producer. After forecasting the next day electricity price $\xi^{\text {est }}$, the producer solves a self-scheduling problem for each generator $i$ :

$$
\begin{aligned}
& \max _{p_{h i}^{\text {est }}, z_{t}^{i}, v_{t}^{i}, y_{t}^{i}} \sum_{h=1}^{H} \xi^{e s t} p_{h i}^{\text {est }}-c_{h}^{i}(x), \\
& \text { subject to }\left(p_{h i}^{e s t}, z_{h}^{i}, v_{h}^{i}, y_{h}^{i}\right) \in \prod^{i}(x)
\end{aligned}
$$

where $\Pi^{i}(x)$ is the feasible operating region for the generator $i$. We model the feasible operation region using a set of mixed linear integer constraints as in [7], which outlines the power output limit, ramping rate, and minimum up and down time, see [7] for the formulations. [8] shows that hedging performance and consequently the income from production strongly depends on the flexibility of its generation facility, i.e., the feasible region described in $\Pi^{i}(x)$. In this self-scheduling problem, $c_{h}^{i}$ is the production cost for the hour $h$ and is composed of the shutdown cost $C^{i}$, start-up cost $S^{i}$, fixed cost $A^{i}$, fuel cost $\zeta_{h}^{i} b^{i} p^{\text {est }}$ and the emission cost $r_{h} e^{i} p^{\text {est }}$, where $b^{i}$ and $e^{i}$ are baseline fuel cost rate and emission rate of the technology $i$ :

$$
c_{h}^{i}=C^{i} z_{h}^{i}+A^{i} v_{h}^{i}+S^{i} y_{h}^{i}+\left(\zeta_{h}^{e s t} b^{i}+r_{h} e^{i}\right) p_{h i}^{e s t} .
$$

$z_{h}^{i}, v_{h}^{i}$ and $y_{h}^{i}$ are the indicator variables corresponding to each of the listed events. In (6), the production cost is linear with the production amount. We also observe that a larger generator costs more to startup and shutdown, and incurs higher fixed cost.

After solving the self-scheduling problem, the producer shall make a bidding combination to maximize its chance of getting the optimal self-scheduling production plan from ISO, see [7] for details on the bidding strategy. The strategy enables the producer to generate the optimal amount from its self-scheduling model with a pre-specified confidence level, provided that the mean and variance of the producer's price forecasting model are correct. On the day of delivery, the producer will re-optimize its production plan based on the bidding result and the current price information:

$$
\begin{aligned}
& \max _{\substack{i \\
z_{h}^{i}, v_{h}, y_{h}^{i}}} \sum_{h=1}^{H} \xi^{\text {com }} p_{h i}^{c o m}-c_{h}^{i}, \\
& \text { subject to }\left(z_{h}^{i}, v_{h}^{i}, y_{h}^{i}\right) \in \prod^{i}(x)
\end{aligned}
$$

where

$$
\left(p_{1 i}^{c o m}, \xi_{1 i}^{c o m}\right), \cdots,\left(p_{H i}^{c o m}, \xi_{H i}^{c o m}\right),
$$

are the bidding result and the cost function

$$
c_{h}^{i}=C^{i} z_{h}^{i}+A^{i} v_{h}^{i}+S^{i} y_{h}^{i}+\left(\zeta_{h}^{t r u} b^{i} i+r_{h} e^{i}\right) p_{h i}^{c o m},
$$

uses the updated price information. Note that the producer only submits feasible bids in (5), hence the problem (6) is always feasible. Hence the true cash flow of the generator $i$ in a portfolio $x$ on day $d$ under scenario $s$ is:

$$
f_{d i}^{s}\left(x_{i}\right)=\sum_{h=1}^{H} \xi^{c o m} p_{h i}^{c o m}-c_{h}^{i}
$$

Note that the relationship between the cash flow $f_{d i}\left(x_{i}\right)$ and a portfolio $x$ is determined by the complicated forecasting and bidding procedures of a power producer. Though it is impossible to write $f_{d i}\left(x_{i}\right)$ as an elementary function of $x$, it is straightforward to simulate it: for a given a portfolio $x$ and a scenario $\eta^{s}=\left(r^{s}, \zeta^{s}, \xi^{s}\right)$, one can mimic the producer's decision process as the following:

1) forecast the electricity and natural gas price to get $\left(\zeta^{\text {est }}, \xi^{e s t}\right)$;

2) solve the self-scheduling problem (5) to get $p^{\text {est }}$;

3) follow the bidding strategy in [7] and return the bidding result $p^{\text {com }}$ using $\xi^{\text {s }}$ as $\xi^{\text {tru }}$;

4) adjust the production plan by solving (7) using $\xi^{s}$ as $\xi^{\text {tru }}$ in computing $c_{h}^{i}$;

5) calculate $f_{d i}^{s}\left(x_{i}\right)$ for all $i \in I$, and let $f_{d}^{s}(x)=\sum_{i \in I} f_{d i}^{s}\left(x_{i}\right)$.

\section{Model the Uncertainties}

\subsection{Electricity Price Uncertainty}

In a day-ahead market, clearing price for each hour could be modeled as a random variable. Especially, the characteristics of seasonality on annual and weekly level, mean- 
reversion and jumps should be appropriately reflected. The infrequent but large jumps caused by extreme load fluctuation such as severe weather, generation outage or transmission failure, make the energy market quite different from a financial market. Though this paper is not a research on modelling electricity price, its importance for this research is evident: only under a properly generated set of pricing scenarios, one can correctly evaluate the performance of a generator portfolio.

There are extensive research on how to generate electricity price scenarios. For example, Markov regime switching model has been proposed in $[9,10]$ develops a pure mean-reversion model with the capability to create jumps; the jump diffusion/mean-reversion model is studied in [11]; neural network is used to predict prices in California in [12]. A comprehensive review article in this field appeared in [13]. Here we use Markov regime switching model due to its flexibility in modelling the jumps.

A jump in electricity price can be considered as a change from the base regime to the spike regime in a two-regime model. The switching itself is modeled as a Markov chain. Let $R_{t}=1$ indicate the base regime, and $R_{t}=2$ indicate the spike regime, the Markov transition matrix $P$ contains the probabilities $p_{i j}$ of switching between the two regimes:

$$
P=\left(\begin{array}{cc}
p_{11} & 1-p_{11} \\
1-p_{22} & p_{22}
\end{array}\right) .
$$

In a Markov process, the probability of regime $j$ at time $t+m$ starting from state $i$ at time $t$ equals $\left\{P^{m}\right\}_{i j}$.

Now we demonstrate how to use Markov switching model in a particular market. Given a set of historical price data $p_{1}, \cdots, p_{7 W}$ for $W$ weeks, the first step is to de-seasonalize the data by fitting a deterministic nonlinear seasonality model [13]:

$$
\begin{aligned}
S_{h}= & \alpha h+\beta_{1} \sin \left(\left(h+\beta_{2}\right) \frac{2 \pi}{24}\right)+\chi D_{h} \\
& +\kappa_{1} \cos \left(\left(h+\kappa_{2}\right) \frac{2 \pi}{8760}\right),
\end{aligned}
$$

where

$$
D_{h}= \begin{cases}1 & \text { if hour } h \text { is in weekend or holiday } \\ 0 & \text { otherwise. }\end{cases}
$$

Here the $\beta_{1}, \beta_{2}$ capture the intra-day change, $\chi$ describes the change of weekends and holidays, and $\kappa_{1}$, $\kappa_{2}$ reflect the annual periodicity.

In a second step, after we get the optimal estimates of parameters $\alpha, \beta_{1}, \beta_{2}, \chi, \kappa_{1}, \kappa_{2}$, we compute the de-seasonalized log-price:

$$
Y_{h}=\log \left(\xi_{h}-S_{h}\right) .
$$

We model the de-seasonalized log-price using the Markov regime switching model [9]. Under this model, one can flexibly choose a stochastic process to describe the log-price in the spike regime, yet another stochastic process to describe the log-price in the base regime. Usually a different stochastic process is chosen to model the jumps in the spike regime. We model the log-price in the base regime as a mean-reversion process, i.e.,

$$
\mathrm{d} Y_{h}=\vartheta\left(\mu_{1}-Y_{h}\right) \mathrm{d} h+\sigma_{1} \mathrm{~d} B_{h} .
$$

The drift term $\vartheta\left(\mu_{1}-Y_{h}\right)$ is negative if the electricity price is higher than the mean $\mu_{1}$ and positive if it is lower. Hence the drift term drags the price back to its mean value $\mu_{1}$ in the long run, with $\vartheta$ describing the speed of reverting to the mean value. The stochastic term $\mathrm{d} B_{h}$ is the increment of the standard Brownian motion, and is multiplied by the market volatility $\sigma_{1}$.

We model the log-price in the spike regime as a lognormally distributed random variable, i.e., $\log \left(Y_{h}\right)$ follows a normal distribution $N\left(\mu_{2}, \sigma_{2}\right)$. The mean $\mu_{2}$ is usually higher than the mean $\mu_{1}$ of the base regime. The $\sigma_{2}$ controls the magnitude of the spikes.

Finally, we estimate the parameters $\left(\vartheta, \mu_{1}, \mu_{2}, \sigma_{1}\right.$, $\left.\sigma_{2}, p_{11}, p_{22}\right)$ using the de-seasonalized historical log-price data. The estimation is quite involved, but is clearly documented in [14]. Using true historical data from a specific market, one may capture the market characteristics: frequency and magnitude of jumps, means and variances of the price in the two regimes, and the speed of mean-reversion in the base regime.

\subsection{Natural Gas Price Uncertainty}

As many countries and regions have planned to abandon the coal generators in near future, natural gas prices become one of the most important factors in deciding the optimal generator portfolio. We model the natural gas price as a Brownian motion with positive drift:

$$
\mathrm{d} \zeta_{d}=\vartheta_{\zeta} \mathrm{d} t+\sigma_{\zeta} \mathrm{d} B_{d}
$$

And we model the coal price as constant since its volatility is relatively low.

\subsection{Carbon Tax Uncertainty}

To correctly evaluate the performance of a generator portfolio, we need a comprehensive set of carbon tax scenarios as well. Carbon tax uncertainty does naturally follow a stochastic process as the natural gas price or the electricity price. Instead it is a complicated movement 
driven by economical and political forces. In a practical implementation, one could follow advices from policy analysts and generate most likely scenarios for the local jurisdiction. In this study, we create the following discrete model for simplicity: 1) there are equal probability of implementing a carbon tax in $T, 2 T, 3 T, 4 T$, $5 T$ years away from now; 2 ) if a carbon policy has not been adopted $i T$ years from now, then the conditional probability of adopting it later is updated to $1 /(5-i)$. Following this conditional probability updating scheme, if a carbon policy has not been implemented $4 T$ years from now, then the chance of implementing it at $5 T$ is one, which respects our believe that a carbon policy will be implemented sooner or later. 3) the carbon tax rate $r$ could be $r_{\text {high }}$ with a probability $p_{\text {high }}$ or $r_{\text {low }}$ with a probability $1-p_{\text {high }}$ for each possible implementation. Once a carbon policy with rate $r$ is implemented, we assume it remains constant in the planning horizon of this study. Figure 1 shows all carbon tax possibilities of this discrete model.

We note that some states/provinces in North America have implemented pilot carbon tax and in Europe the carbon emission is traded in the $\mathrm{CO}_{2}$ market; however, carbon policy is evolving and the associated uncertainty remains a big factor in optimal generator portfolio problem.

To generate a scenario $\eta^{s}=\left(r^{s}, \zeta^{s}, \xi^{s}\right)$, one follows the models in 4.3, 4.2 and 4.1 separately. This implicitly assumes independence among $(r, \zeta, \xi)$. We acknowledge that the possible increase of electricity price due to a change in fuel price can only be captured by a very complicated macro-economic model, which is out of the scope of this paper. However, it seems evident that the technological substitution has more elasticity than the electricity demand, which justifies our approach as we focus on the dynamics of the optimal composition of technologies under the changing carbon tax and fuel prices.

\section{Computational Tractability}

The optimal generator portfolio decision model (4) with constraints (1) and (2) is not an usually mathematical programming model since $f_{d}(x)$ can only be evaluated by steps (1)-(5) in the Section 3. A natural choice is to conduct a simulation optimization research, where we equip a power producer with a generator portfolio $x^{0}$, and let it act as a smart agent following steps (1)-(5) in the Section 3. At end of such a simulation, one get a corresponding NPV for the generator portfolio $x^{0}$. To get an expected NPV for the portfolio $x^{0}$, one repeats the process for each scenario in a large sample

$\left\{\eta^{s}, s=1, \cdots, S\right\}$. A simple simulation optimization algorithm calculates the $\operatorname{ENPV}\left(x^{i}\right)$ for many different generator portfolios $x^{i}, i=1, \cdots, N$ (tries), and at end picks the portfolio with the highest ENPV. A sophisticated simulation optimization algorithm may conduct more intelligent search for better $x^{i}$, see [15] for such an advanced searching algorithm. $N$ needs to be large for a simple simulation optimization procedure to ensure sufficient coverage. $N$ does not need to be huge for a sophisticated procedure, however it is non-deterministic in general. For both cases, optimality of $x$ can not be guaranteed. The computational burden is prohibitive as we need to solve the producer's self-scheduling problem (5) for $D$ times for each scenario $\eta^{s}$ and portfolio $x^{i}$, which brings the total to $D \times S \times N$. Considering a 30 year problem with $D=10950$, a moderate number of scenarios $S=26$, and $N=1000$ tries, the total number of self-scheduling problem to be solved is $284,700,000$. We note that each (5) is a hard mixed integer linear programming (MIP) problem, and we haven't counted the adjusting problem (7) and other steps listed in the section 3 yet. This computational burden makes the optimal generator portfolio problem with daily bidding details practically intractable.

To overcome this difficulty, we prove a useful theorem, which allows us to evaluate $f_{d i}^{s}(\mathbf{1})$ for $d \in D$, $i \backslash$ in $I, s \in S$ only once; and find an optimal generator portfolio $x^{*}$ by solving a simple linear programming model.

Theorem 5.1 (Decomposition theorem) The cash flow of a generator portfolio depends on the capacity of individual generators linearly, i.e.,

$$
f_{d}^{s}(x)=\sum_{i \in I} f_{d i}^{s}(1) x_{i} .
$$

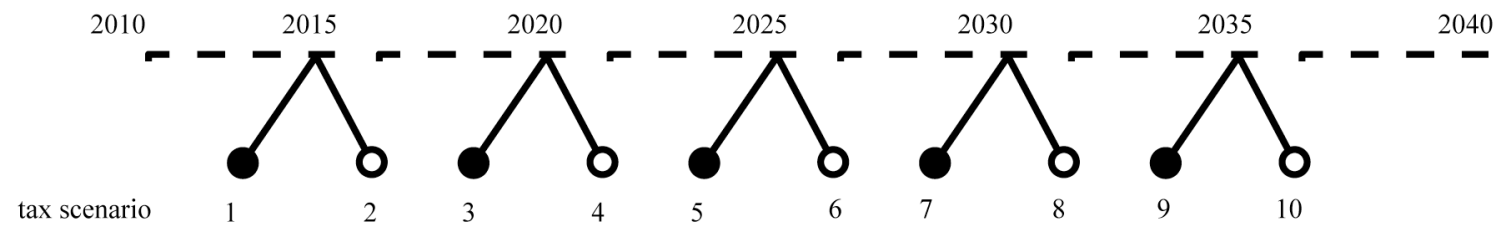

Figure 1. The diagram shows two possible outcomes of carbon tax rate (high or low) at each of the five calendar years shown in the graph, which brings the total number of carbon tax scenarios to ten in this study. A filled circle represents the event that a low tax rate is implemented in the associated year. 
Proof. As shown in [7], in the price-taker bidding strategy, the performance of each generator in a portfolio can be evaluated independently, i.e.,

$$
f_{d}^{s}(x)=\sum_{i \in I} f_{d i}^{s} x_{i} .
$$

It remains to show that $f_{d i}^{s}\left(x^{i}\right)=f_{d i}^{x}(1) x_{i}$. The conclusion follows the linearity of the objective function and the feasible region in producer's bidding step (5), adjusting step (9) and the cash flow formula (9).

Following Theorem 5.1, one only needs to evaluate $f_{d i}^{s}(1)$ for a "standard" generator with capacity 1 , and compute $g_{i}=\sum_{d \in D} \sum_{s \in S} f_{d i}^{s}(1) \cdot \gamma^{d}$ and $g_{m i}^{s}=\sum_{d \in M_{m}} f_{d i}^{s}(1)$. Then the optimal generator portfolio model (4), subject to constraints (1) and (2) is equivalent to the following linear programming problem:

$$
\begin{aligned}
\max _{x} & \sum_{i \in I} g_{i}\left(x_{i} / k_{i}\right) \\
\text { s.t. } & \sum_{i \in I} x_{i} \leq 1 \\
& \sum_{i \in I} g_{m i}^{s}\left(x_{i} / k_{i}\right) \geq \bar{f}_{m}, \forall s \in S, m \in M
\end{aligned}
$$

In the above model, $x_{i} / k_{i}$ gives the capacity of generator type $i$ for $x_{i}$ amount of investment.

\section{Case Study}

We use the historical hourly electricity price data of the Independent Electricity System Operator of Ontario from February 18th 2009 to April 2nd 2010 to fit the Markov regime switching model, and the fitted parameters are shown in the Tables $\mathbf{1}$ and $\mathbf{2}$. We generate the natural gas price scenarios following the equation (12). The carbon policy scenarios are generated from the discrete model in subsection 4.3 with the five discrete years being 2015, 2020, 2025, 2030, and 2040. We let $p_{\text {high }}=2 / 3$, $r_{\text {high }}=\$ 200 / t C$ and $r_{\text {high }}=\$ 50 t C$. A total of 26 identical and independently distributed (i.i.d.) samples of $\eta^{s}=\left(r^{s}, \zeta^{s}, \xi^{s}\right)$ are generated. Two electricity price sample paths are shown in Figure 2.

We use three types of thermal generators $C, G, T$ in this study. The technological parameters, compiled

Table 1. De-seasonality parameters.

\begin{tabular}{cccccc}
\hline$\alpha$ & $\beta_{1}$ & $\beta_{2}$ & $\gamma$ & $\kappa_{1}$ & $\kappa_{2}$ \\
\hline 0.00012 & 17.36 & -33.13 & 7.87 & -2.38 & -33543 \\
\hline
\end{tabular}

Table 2. Markov switching model parameters.

\begin{tabular}{ccccccc}
\hline$\vartheta$ & $\mu_{1}$ & $\mu_{2}$ & $\sigma_{1}$ & $\sigma_{2}$ & $p_{11}$ & $p_{22}$ \\
\hline 0.0471 & 3.48 & 1.51 & 0.03 & 0.139 & 0.09 & 0.41 \\
\hline
\end{tabular}

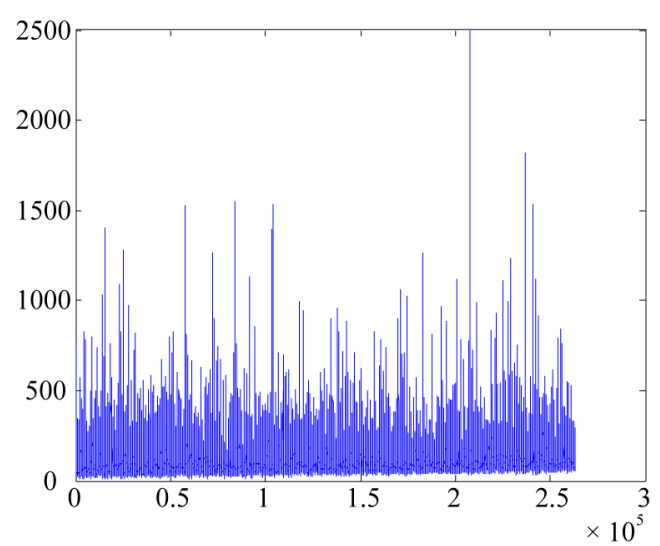

(a)

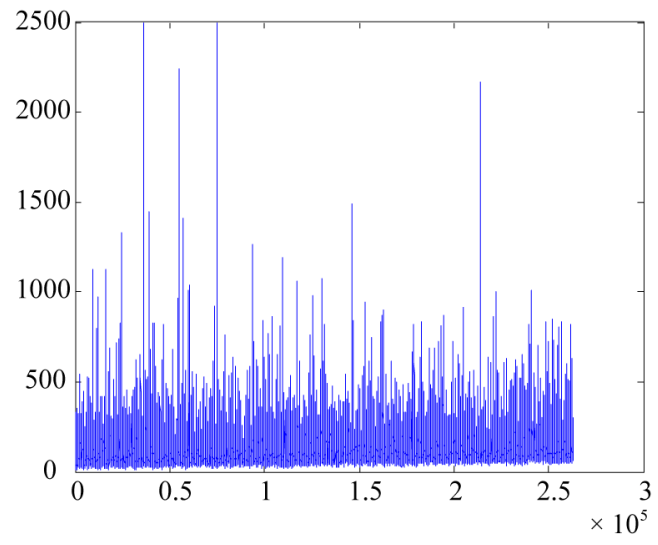

(b)

Figure 2. Two scenarios of hourly Ontario Electricity Price for 30 years. $x$-axis represents number of days, and $y$-axis indicates the electricity price. (a) Scenario a; (b) Scenario b.

from [16,17], are representative for a typical coal generator, a natural gas generator and a combined gas turbine. In Table 3, we list the following technical parameters: minimum power output $\underline{P}$, maximum power output $\bar{P}$, start-up ramp limit $S U$, shutdown ramp limit $S D$, ramp-up rate limit $R U$, ramp-down rate limit $R D$, minimum up time $U T$ and minimum down time $D T$. In Table 4, we give the following cost parameters: capital investment per unit of capacity $k^{i}$, fixed operating cost $A^{i}$, start-up cost $S^{i}$, shutdown cost $C^{i}$, baseline fuel cost $b^{i}$ (at current natural gas price $\$ 8 / \mathrm{GJ}$ ), emission rate $e^{i}$. Note that the baseline fuel cost implicitly

Table 3. Generator technological parameters.

\begin{tabular}{ccccccccc}
\hline & $\underline{P}$ & $\bar{P}$ & $S U$ & $S D$ & $R U$ & $R D$ & $U T$ & $D T$ \\
\hline & $\mathrm{MW}$ & $\mathrm{MW}$ & $\mathrm{MW} / \mathrm{h}$ & $\mathrm{MW} / \mathrm{h}$ & $\mathrm{MW} / \mathrm{h}$ & $\mathrm{MW} / \mathrm{h}$ & $\mathrm{h}$ & $\mathrm{h}$ \\
$\mathrm{C}$ & 200 & 430 & 80 & 70 & 30 & 20 & 23 & 23 \\
$\mathrm{G}$ & 112 & 294 & 170 & 160 & 80 & 70 & 4 & 4 \\
$\mathrm{~T}$ & 180 & 450 & 155 & 134 & 76 & 59 & 8 & 7 \\
\hline
\end{tabular}


Table 4. Generator economic parameters.

\begin{tabular}{ccccccc}
\hline & Capital & Fixed & Starup & Shutdown & Fuel & Emission \\
\hline & $\$ / \mathrm{kW}$ & $\$ / \mathrm{kW}$ & $\$$ & $\$$ & $\$ / \mathrm{kW}$ & $\mathrm{kg}-\mathrm{C} / \mathrm{MWhr}$ \\
$\mathrm{C}$ & 1200 & 22 & 1038 & 56 & 5 & 228 \\
$\mathrm{G}$ & 450 & 15 & 230 & 21 & 10 & 89 \\
$\mathrm{~T}$ & 1550 & 26 & 549 & 40 & 9 & 27 \\
\hline
\end{tabular}

reflects the thermal efficiency of a generator. To get the fuel cost rate for generator $i$ at $h$, one just need to multiply $\zeta_{h} / 8$ with the corresponding baseline fuel cost.

We let the natural gas price $\zeta_{1}=\$ 8 / G J$, $\vartheta=\$ 1.2 \times 10^{-5} / G J, \quad \sigma_{\zeta}=\$ 2.1 \times 10^{-4} / G J$, which approximate the current market price and the hourly drifting rate.

We set the minimum cash flow $\bar{f}_{m}=30$ for each month. In practice, this minimum profit level should be set according to the producer's business and financial status. Also note that the constraint (2) could be read as if the portfolio worths $\$ 1$ investment, hence the monthly minimum cash flow level should be set for this $\$ 1$ portfolio.

The computation is conducted on a personal computer with $2.13 \mathrm{GHZ}$ Intel Core 2 Duo and 2 GB $800 \mathrm{MHZ}$ DDR2 SDRAM. We used MATLAB to generate scenarios, fit parameters and evaluate $f_{d i}^{s}$, and used GLPK/ GMPL for modelling the self-scheduling problem (7), adjusting problem (5) and the portfolio selection problem (P). Most tasks took negligible time, except evaluating $f_{d i}^{s}(1)$ for the 26 scenarios, 3 generators and 10950 days, which requires solving 1,708,200 MIP problems, see (7) and (5). These problems are not related and can be solved in parallel. A simple serial code written in ANSI $\mathrm{C}$ solved all these MIP problems in 9 hours. The portfolio selection problem $(\mathrm{P})$ has three decision variables, one budget constraint (1), and 9360 minimal monthly cash flow constraints (2), is solved to optimality in three seconds. In the contrast, a simple simulation optimization method with 1000 tries, would need 9000 hours to deliver a suboptimal result.

The optimal solution $x_{i}^{*}$ from our linear programming model $(\mathrm{P})$ is:

- $C=0 \%, G=8 \%, T=92 \%$ in terms of capital value; or

- $C=0 \%, G=23 \%, T=77 \%$ in terms of capacity,

where $C$ stands for carbon, $G$ for natural gas and $T$ for combined gas turbine.

The result clearly shows that though coal generator technology has lowest production cost, the prohibitive environmental fee in some high carbon tax scenarios makes it unfavourable. If one assumes that high carbon tax has a high possibility as this model does, then the coal generators should not show up in an optimal portfolio. The combined gas turbine and natural gas generator exhibit complimentary features under different scenarios, and the optimal solution recommends the 23/77 combination. The result might change if a drastically different carbon tax model is applied. A decision maker may supply the model with a different perspective of the carbon tax policy and gain a different optimal portfolio.

The daily simulation for a "standard" generator over 30 years also reveals some insightful phenomena. We show two of such simulations in 1 . The subgraph (a) shows a scenario where the high carbon tax is implemented in 2015; and the subgraph (b) shows a scenario where a low carbon tax is implemented in 2025 . We first observe that the order of performance of the three generator technologies could change significantly after the carbon tax. The coal technology (blue line) clearly outperforms the other two technologies before the carbon tax, but after the carbon tax, it yields negative cash flow in scenario (a) and ranked in a second place in the scenarion (b). Secondly, many downward cashflow jumps dominates both subgraphs (a) and (b). A careful drill down analysis shows that these are caused by inaccurate electricity price forecast. Historical electricity price also shows many jumps due to many unpredictable events including network congestion. The Markov regime switching model can generate these jumps, thus mimicking the price pattern. However, forecasting these jumps at daily level can only be accurate in a statistical sense at the best, hence a power producer's daily forecast error is unavoidable. As a result the submitted bids could be far off the true optimal production plan, hence the flexibility of adjusting the production plan is necessary. This phenomena further emphasize the importance of studying the long term planning problem with the daily bidding details. Without this "nano" level investigation, a significant profit loss due to the unavoidable price jump cannot be correctly captured and evaluated. Finally, we note that natural gas generator has less downward cash flow jumps, and the occurred jumps are small in magnitude. We contribute this to the technology's flexibility. In our drill down analysis, the natural gas generator technology allows more flexible production adjustment when the forecast is not accurate. As shown in the Figure 3, this flexibility shapes the cash flow pattern $f_{d i}^{s}$ of different technology $i$ drastically, and yields tremendous loss savings in the planning horizon under study.

\section{Conclusions}

Though it is widely accepted that the market deregulation and the evolving carbon tax have profound impact on an optimal generator portfolio, it is challenging for 


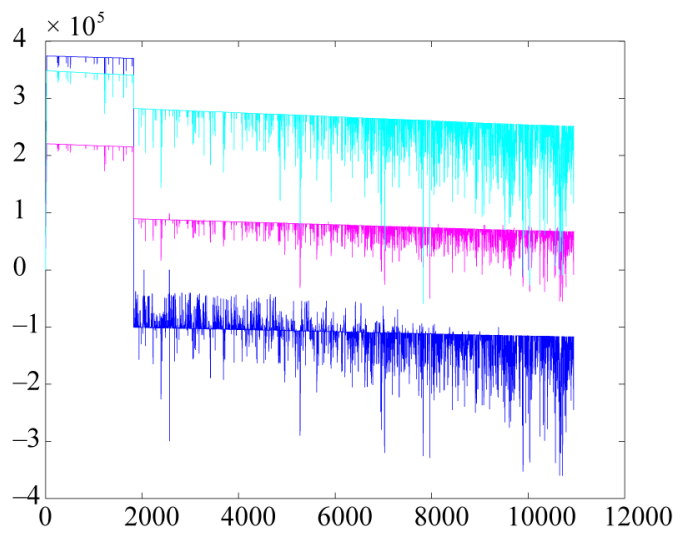

(a)

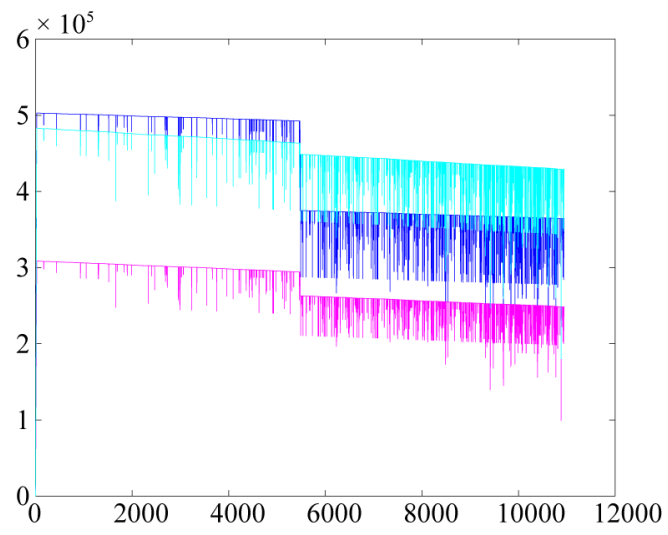

(b)

Figure 3. $x$-axis shows days $d$ and the $y$-axis shows cash flow $f_{d i}(1)$. Blue line for the coal generator, magenta line for natural gas, and cyan line for the combined gas turbin. (a) High carbon tax since 2015; (b) Low carbon tax since 2025.

senior executives to make a quantitative decision analysis, i.e., what's the optimal percentage for each generator technology? The proposed model incorporates into consideration the important day-ahead bidding activity, and three major uncertainty sources underlying the decision problem. For such a long term planning study, these meticulous considerations at daily operation level usually lead to computational intractability. We overcome this difficulty by vertically decomposing the problem into individual "standard" generator of capacity one, and convert the problem into a simple linear programming model. We are able to compute the proposed model on a personal computer within a reasonable time limit. The result shows that the daily negative cash flow jumps are dominating, and constitute a significant part in evaluating the ENPV of a portfolio. Once supplied with the market and candidate generator parameters, together with the carbon tax scenarios of the local jurisdiction, a decision maker can apply the model and the decomposition algorithm to find an optimal generator portfolio efficiently.

\section{References}

[1] J. M. Arroyo and A. J. Conejo, "Optimal Response of a Thermal Unit to an Electricity Spot Market," IEEE Transactions on Power Systems, Vol. 15, No. 3, 2000, pp. 1098-1104. doi:10.1109/59.871739

[2] ISO New England Inc., Technical Report, 2002. http://www.iso-ne.com

[3] ERCOT, The Electric Reliability Council of Texas, Inc., Technical Report, 2002. http://www.ercot.com

[4] J. Hinz, "Optimizing a Portfolio of Power-Producing Plants,” Bernoulli, Vol. 9, No. 4, 2003, pp. 659-669. doi:10.3150/bj/1066223273

[5] N. M. Pindoriya, S. N. Singh and S. K. Singh, "Optimal Generation Portfolio Allocation in Competitive Electricity Market," Annual IEEE India Conference, Gujarat, 18-20 December 2009, pp. 1-4. doi:10.1109/INDCON.2009.5409351

[6] X. Yin, Z. Y. Dong and T. K. Shaha, "Optimal Portfolio Selection for Generators in the Electricity Market," Proceeding of IEEE PES General Meeting, Pittsburgh, 20-24 July 2008, pp. 1-7.

[7] A. J. Conejo, F. J. Nogales and J. M. Arroyo, "PriceTaker Bidding Strategy under Price Uncertainty," IEEE Transactions on Power Systems, Vol. 17, No. 4, 2002, pp. 1081-1087. doi:10.1109/TPWRS.2002.804948

[8] J. Doege, P. Schiltknecht and H.-J. Lu"thi, "Risk Management of Power Portfolios and Valuation of Flexibility," OR Spectrum, Vol. 28, No. 2, 2006, pp. 267-287. doi:10.1007/s00291-005-0005-4

[9] M. Bierbrauer, S. Tru"ck1, and R. Weron, "Modeling Electricity Prices with Regime Switching Models," Lecture Notes in Computer Science, Vol. 3039, 2004, pp. 859-867. doi:10.1007/978-3-540-25944-2 111

[10] M. T. Barlow, "A Diffusion Model for Electricity Prices," Mathematical Finance, Vol. 12, No. 4, 2002, pp. 287-298. doi:10.1111/j.1467-9965.2002.tb00125.x

[11] P. Skantze, M. Ilic and J. Chapman, "Stochastic Modeling of Electric Power Prices in a Multi-Market Environment," Proceeding of Power Engineering Society Winter Meeting, Singapore, 23-27 January 2000, pp. 1109-1114.

[12] F. Gao, X. Guan, X.-R. Cao and A. Papalexopoulos, "Forecasting Power Market Clearing Price and Quantity Using a Neural Network Method," Proceeding of Power Engineering Society Summer Meeting, Seattle, 16-20 July 2000, pp. 2183-2188.

[13] J. J. Lucia and E. S. Schwartz, "Electricity Prices and Power Derivatives: Evidence from the Nordic Power Exchange," Review of Derivatives Research, Vol. 5, No. 1, 2002, pp. 5-50. doi:10.1023/A:1013846631785

[14] J. D. Hamilton, "Regime Switching Models," Palgrave Dictionary of Economics, Palgrave McMillan Ltd., New York, 2005. 
[15] L. J. Hong and B. L. Nelson, "Discrete Optimization via Simulation Using COMPASS," Operations Research, Vol. 54, No. 1, 2006, pp. 115-129. doi:10.1287/opre.1050.0237

[16] C. Wang and S. M. Shahidehpour, "Ramprate Limits in Unit Commitment and Economic Dispatch Incorporating Rotor Fatigue Effect," IEEE Transactions on Power Sys- tems, Vol. 9, No. 3, 1994, pp. 1539-1545. doi:10.1109/59.336106

[17] P. S. Reinelt and D. W. Keith, "Carbon Capture Retrofits and the Cost of Regulatory Uncertainty," The Energy Journal, Vol. 28, No. 4, 2007, pp. 101-128. doi:10.5547/ISSN0195-6574-EJ-Vol28-No4-5 